\title{
CONTRIBUIÇÃO DA ESTRUTURA INTERNA NA PERDA DE CARGA DE FILTROS DE AREIA UTILIZADOS NA IRRIGAÇÃO
}

\author{
MARCIO MESQUITA ${ }^{1}$, ROBERTO TESTEZLAF ${ }^{2}$, JUAN C. SALCEDO ${ }^{3}$, \\ DOUGLAS R. BIZARI ${ }^{4}$
}

\begin{abstract}
RESUMO: A dinâmica de operação dos filtros de areia afeta o desempenho hidráulico do sistema de irrigação, elevando a perda de carga e alterando a altura manométrica total do sistema. Buscando entender parte dessa dinâmica, o objetivo deste trabalho foi determinar o efeito da estrutura interna de filtros de areia na perda de carga de três modelos de equipamentos fabricados no Brasil, sem a presença do elemento filtrante e utilizando água limpa. Adicionalmente, com o ajuste do modelo matemático exponencial aos dados experimentais, procurou-se estabelecer comparações entre os tipos de estrutura dos filtros avaliados. Os ensaios foram realizados em um módulo experimental construído no Laboratório de Hidráulica e Irrigação da FEAGRI/UNICAMP. Os resultados mostraram que a estrutura hidráulica interna dos filtros determinou comportamentos hidráulicos diferenciados e que os tipos de estruturas (placa difusora e drenos) alteraram o padrão de operação dos modelos ensaiados. A função matemática proposta representou, significativamente, o fenômeno físico de perda de carga para as condições do experimento.
\end{abstract}

PALAVRAS-CHAVE: irrigação localizada, placa difusora, drenos.

\section{CONTRIBUTION OF THE INTERNAL STRUCTURE IN THE SAND FILTERS HEAD LOSS USED IN IRRIGATION}

\begin{abstract}
The dynamic of operation of sand filters affect the hydraulic performance of irrigation systems, increasing the head loss and changing the total dynamic head of the system. Trying to understand part of this dynamic, the objective of this research was to determine the effect of internal hydraulic structures in the head loss of three sand filters of commercial models, manufactured in Brazil and operating without the filter layer and with clean water. In addition, using an exponential mathematical model adjusted to the experimental data, comparisons among types of structure of each evaluated filter were performed. The trails were accomplished in the experimental module developed in the Laboratory of Hydraulics and Irrigation of FEAGRI / UNICAMP. The results showed that the filters structures determined differentiate hydraulic behaviors and each type of structures (diffuser plate and drains) change the standard operation for the evaluated filters. The mathematical function proposed represented significantly the head loss physical phenomenon for the experimental conditions.
\end{abstract}

KEYWORDS: localized irrigation, diffuser plate, drains.

\footnotetext{
${ }^{1}$ Eng $^{\circ}$ Agrícola, Doutorando, Faculdade Engenharia Agrícola - UNICAMP, Campinas - SP, marcio.mesquita@ feagri.unicamp.br.

${ }^{2}$ Eng $^{\circ}$ Agrícola, Professor Titular, Faculdade de Engenharia Agrícola - UNICAMP, Campinas - SP, bob@ feagri.unicamp.br.

${ }^{3}$ Eng $^{\mathrm{O}}$ Agrícola, Mestre, Faculdade Engenharia Agrícola - UNICAMP, Campinas - SP, camilosalcedo2003@ yahoo.es.

${ }^{4}$ Eng $^{\mathrm{O}}$ Agrônomo, Doutor, Faculdade Engenharia Agrícola - UNICAMP, Campinas - SP, douglasbizari@ yahoo.com.br.

Recebido pelo Conselho Editorial em: 19-4-2010

Aprovado pelo Conselho Editorial em: 21-10-2011
} 


\section{INTRODUÇÃO}

Os filtros de areia são equipamentos que funcionam atendendo a diversos fundamentos da mecânica dos fluidos, sendo necessário o conhecimento do efeito dos componentes estruturais sobre o seu funcionamento para permitir a definição correta dos parâmetros de projeto e de sua operação em campo.

Os elementos estruturais internos mais importantes, pertencentes aos filtros de areia, são: placa difusora e drenos. O primeiro determina as condições de movimento do afluente sobre o leito filtrante, e o segundo proporciona condições ideais para a limpeza da camada de areia e a condução do efluente sem o arraste de impurezas e/ou material filtrante para o sistema de irrigação.

A placa difusora é responsável por diminuir a energia cinética do escoamento de água produzida pelo bombeamento, transformando-a em energia potencial e, assim, distribuir o fluxo de água uniformemente sobre o leito filtrante e evitar a movimentação de sua camada superficial. BURT (1994) afirma que os difusores internos não são indicados para dispersar altas vazões, recomendando o valor máximo de $61 \mathrm{~m}^{3} \mathrm{~h}^{-1} \mathrm{~m}^{-2}$ para a taxa de filtração, sendo que valores superiores determinariam alterações na superfície da camada filtrante. Dessa forma, o mau dimensionamento e inadequações do projeto estrutural desse acessório podem causar movimentos na superfície do leito filtrante, prejudicando o desempenho hidráulico dos filtros. Se o fluxo de entrada de água é direcionado predominantemente para as paredes do filtro, ocorre intensificação da turbulência do fluido sobre a superfície filtrante, movimentando as partículas de areia dessas regiões para o centro do leito, criando caminhos preferenciais para a filtragem. Esses caminhos diminuem a área efetiva e a altura da camada de filtragem, fazendo que a contaminação do leito por impurezas ocorra rapidamente, reduzindo a eficiência de remoção do filtro e, consequentemente, aumentando a frequência de retrolavagem dos filtros (VERMEREIN \& JOBLING, 1984; BURT, 1994; PHILLIPS, 1995; BENHAM \& ROSS, 2002; MESQUITA, 2010).

Os drenos ou crepinas são responsáveis por determinar maior efetividade da camada filtrante, aumentando seu volume disponível para a filtração, e por permitir a passagem de água filtrada para o sistema de irrigação, impedindo a passagem de grãos da camada filtrante para o seu interior, e por distribuir, uniformemente, o fluxo contrário no leito, durante o processo de retrolavagem. Esse acessório apresenta diferentes formatos e dimensões, sendo o seu posicionamento e número de elementos definidos por critérios dos fabricantes. Um dos problemas que esse sistema pode apresentar é sua ruptura física do elemento, causada pelo seu entupimento e crescimento da pressão interna de filtragem. Essa ruptura determina redução no diferencial de pressão no filtro e a passagem de impurezas para o sistema de irrigação.

A operação adequada de um filtro de areia depende da estimativa correta da sua perda de carga característica, que é causada pela passagem da água de irrigação pelo seu interior, resultante tanto da resistência das partículas do meio filtrante ao movimento do fluído como das perdas de atrito resultante da estrutura hidráulica dos filtros. A perda de carga correspondente à estrutura desse equipamento é uma combinação do efeito do corpo do filtro e das suas peças internas (placa difusora e drenos). O conhecimento da contribuição da estrutura nessas perdas é básico para o projeto de operação dos filtros, pois é preciso estimar o quanto de perda de carga total admissível estará disponível para o processo de retenção de partículas no filtro, possibilitando o correto dimensionamento desses equipamentos (CHANG et al., 1999; TESTEZLAF, 2008).

Apesar da importância desses componentes na dinâmica dos processos hidráulicos de filtragem e retrolavagem, não há evidências técnicas e científicas da existência de desenvolvimento do projeto estrutural por parte dos fabricantes nacionais de filtros, pois as peças presentes nos equipamentos são, na maioria dos casos, adaptações de modelos de filtros importados ou resultados da reutilização de material no processo de fabricação da estrutura do equipamento. A busca por informações técnico-científicas que aprofundem os conhecimentos sobre a influência da estrutura interna dos filtros de areia contribuirá para o desenvolvimento dos projetos desses componentes e na operação e dimensionamento desses equipamentos. 
Visando a contribuir com informações sobre a otimização do projeto e operação de filtros de areia na área agrícola, o objetivo deste trabalho foi determinar o efeito da estrutura hidráulica interna na perda de carga de três modelos de filtros de areia fabricados no Brasil, sem a presença do material filtrante e utilizando água limpa.

\section{MATERIAL E MÉTODOS}

Os ensaios de caracterização hidráulica dos filtros de areia foram realizados no Laboratório de Hidráulica e Irrigação da Faculdade de Engenharia Agrícola/FEAGRI, da Universidade Estadual de Campinas/UNICAMP, no período de 02/2009 a 09/2009.

\section{Módulo hidráulico}

Para a realização dos ensaios, projetou-se e construiu-se um módulo experimental que foi acoplado ao circuito fechado de tubulações presente na estrutura do laboratório, como mostra o esquema da Figura 1.

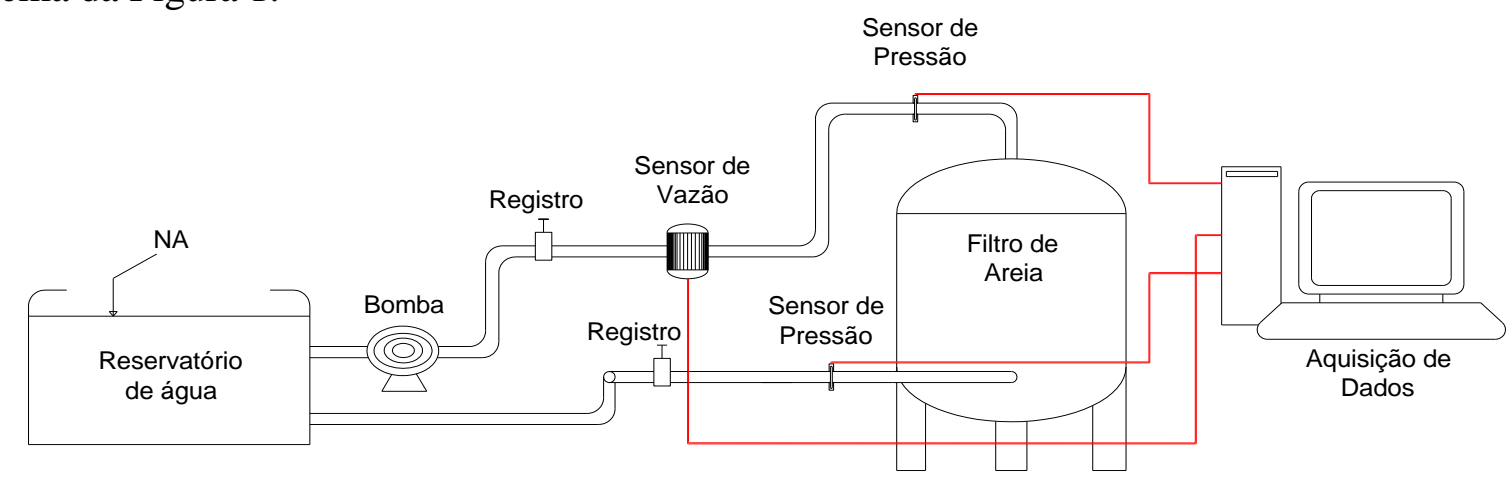

FIGURA 1. Esquema do módulo experimental com detalhes da conexão do sistema de aquisição de dados. Experimental module layout with connection details of the data acquisition system.

O módulo experimental foi instrumentalizado para permitir a aquisição de dados em tempo real, sendo constituído de um sensor de vazão, Marca Seginet, modelo 2551, sinal de saída analógico variando de 4-20 mA, e transdutores de pressão, Marca Motorola, modelo MPX 5700DP cuja faixa de funcionamento é de 0 a $700 \mathrm{kPa}$ e sinal de saída entre $0,2 \mathrm{~V}$ e 4,7 V, acoplados a uma placa de aquisição de dados da Marca National Instruments, PCI 6221, instalada em um microcomputador que tinha a função de controlador. A conversão das leituras analógicas para digital foi realizada pela placa de aquisição de dados gerenciada por uma interface computacional desenvolvida no software Labview. Como os sinais do medidor de vazão eram emitidos em corrente alternada, realizou-se a conversão para voltagem, adequando-os aos requerimentos de entrada da placa de aquisição.

Para a conexão dos transdutores de pressão à tubulação, foram construídas tomadas de pressão do tipo integral e instaladas, atendendo à norma ASABE (1994), as quais permitiam a determinação da pressão a partir de quatro pontos ao longo do mesmo diâmetro da tubulação, aumentando a exatidão da leitura dessa variável.

\section{Condições experimentais}

Foram selecionados três filtros de areia fabricados no Brasil, que tinham a maior participação no mercado nacional, e que se caracterizavam por possuir diferentes componentes internos, denominados neste trabalho como F1, F2 e F3, caracterizados na Tabela 1. 
TABELA 1. Dimensões e características estruturais dos filtros avaliados. Dimensions and structural characteristics of the evaluated filters.

\begin{tabular}{ccccccccc}
\hline \multirow{2}{*}{ Filtro } & \multicolumn{7}{c}{ Características Estruturais } \\
\cline { 2 - 8 } & Modelo & Diâmetro $(\mathrm{cm})$ & Tipo de Crenos & Tipo de Difusor & $\mathrm{n}^{\mathrm{o}}$ Crepina & $\mathrm{H}^{1}$ & $\mathrm{P}_{\mathrm{m}}{ }^{2}$ & $\mathrm{Q}^{3}$ \\
\hline F1 & 15165 & 75 & Braço coletor & Plano c/ borda & 22 & 50 & 7 & 50 \\
F2 & FA07 & 40 & Crepina cilíndrica & Plano & 4 & 60 & 7 & 21 \\
F3 & 520 & 50 & Crepina cônica & Plano c/duplo & 8 & 50 & 10 & 18 \\
\hline
\end{tabular}

$\mathrm{H}^{1}$ :altura do corpo dos filtros em $\mathrm{cm} ; \mathrm{P}_{\mathrm{m}}{ }^{2}$ : pressão máxima de serviço em $\mathrm{kgf.} \mathrm{cm}^{-2} ; \mathrm{Q}^{3}$ : vazão recomendada pelo fabricante, $\mathrm{m}^{3} \cdot \mathrm{h}^{-1}$. Fonte: Catálogos dos fabricantes.

Os detalhes dos sistemas de drenos e do difusor dos filtros avaliados estão apresentados nas Figuras 2 e 3, respectivamente.

$\mathrm{a}$

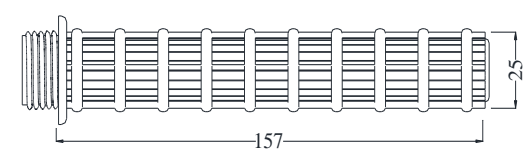

$\mathrm{b}$

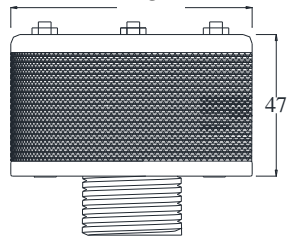

$\mathrm{c}$

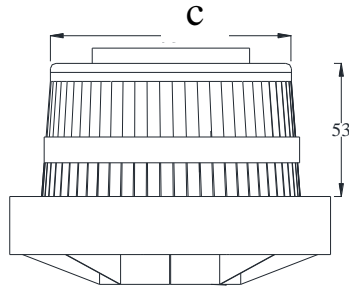

FIGURA 2. Sistemas de drenos dos filtros de areia ensaiados: (a) braço coletor - F1; (b) crepinas cilíndricas - F2 e (c) crepinas cônicas - F3 (unidades em mm). Drain systems of the evaluated sand filters: (a) manifold underdrain - F1 (b) cylindrical drain - F2 (c) conical drain - F3 (units in mm).
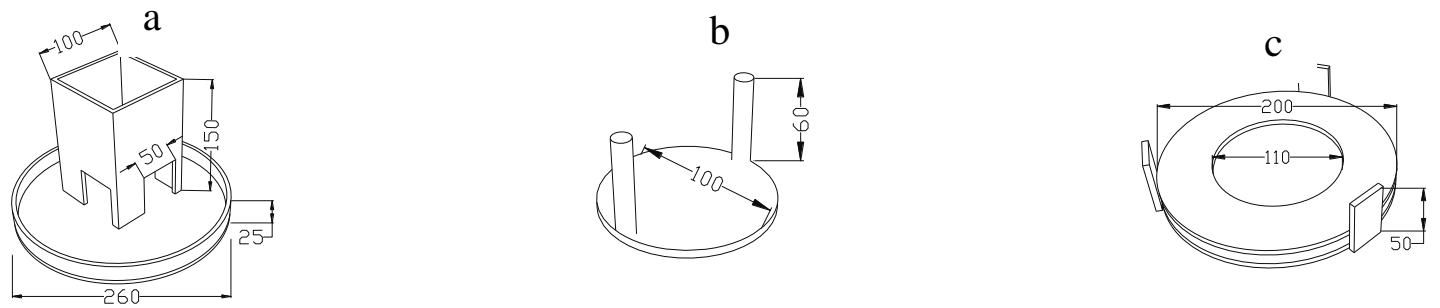

FIGURA 3. Modelos de difusores dos filtros de areia ensaiados: (a) modelos planos com bordas F1; (b) planos - F2 e (c) planos com anel superior - F3 (unidades em mm). Models of diffuser plates of the evaluated sand filters: (a) flat models with edges - F1 (b), simple flat - F2 (c) double flat with top ring - F3 (units in mm).

\section{Procedimento experimental}

A determinação da perda de carga dos modelos de filtro foi realizada para a condição sem material filtrante, ou seja, na ausência da areia em seu interior ou vazio e para a condição de água limpa, $0,142 \mathrm{mg} \mathrm{L}^{-1}$ de concentração média de sólidos suspensos. A leitura de cada variável monitorada (vazão e pressão) tinha duração de três minutos, com o registro de um valor médio por segundo, totalizando 180 valores por média. A partir dos valores médios registrados, determinou-se a perda de carga característica de cada filtro, pela diferença da pressão de entrada e de saída, para uma determinada taxa de filtração. Para cada ensaio, procedeu-se a três repetições, aplicando-se cinco valores de vazão na ordem ascendente, descendente e aleatória, utilizando-se das taxas de filtração de 20; 40; 60; 80 e $100 \mathrm{~m}^{3} \mathrm{~m}^{-2} \mathrm{~h}^{-1}$.

Os valores de taxa de filtração apresentados foram adotados, buscando atender aos intervalos de operação recomendados por diversos autores, e, principalmente, por não haver um consenso 
referente a um padrão recomendado na operação de filtragem. Dessa forma, o intervalo sugerido para os ensaios experimentais abrange as recomendações encontradas na literatura (VERMEREIN \& JOBLING, 1984; ASAE, 1993; PHILLIPS, 1995; PIZARRO CABELO, 1996; BENHAM \& ROSS, 2002).

\section{Análise dos resultados}

Utilizando os valores do diferencial de pressão entre a entrada e a saída dos filtros e os correspondentes valores de taxa de filtração, construíram-se tabelas e gráficos que permitissem a análise do processo.

Buscando caracterizar o comportamento hidráulico dos filtros de areia durante a filtragem com água limpa e que atingisse um valor significativo de correlação entre as variáveis, realizou-se uma simulação matemática, ajustando os valores experimentais de perda de carga em função da vazão do sistema, utilizando a função exponencial (eq.(1)) e procedendo à análise de variância dos resultados. Esse modelo foi escolhido baseado na literatura (ADIN \& ALON, 1986; TESTEZLAF \& RAMOS, 1995; PUIG-BARGUÉS et al., 2005; OLIVEIRA et al., 2007), que afirma a dificuldade de se utilizar a teoria de perda de carga localizada em peças especiais, como filtros, e orienta a não aplicação de equações gerais nesses casos, em virtude de suas próprias características e de fenômenos observados em seu interior, quando da passagem do líquido.

$$
\mathrm{H}_{\mathrm{f}}=\beta_{0} \mathrm{e}^{\beta 1 \mathrm{Q}}
$$

em que,

Hf -perda de carga unitária da estrutura do filtro, $\mathrm{kPa}$;

$\beta_{0}$ - coeficiente de ajuste linear;

$\beta_{1}$ - coeficiente de ajuste exponencial, e

$\mathrm{Q}$ - vazão de filtragem, $\mathrm{m}^{3} \mathrm{~h}^{-1}$.

\section{RESULTADOS E DISCUSSÃO}

A partir dos valores médios de perda de carga em função da taxa de filtração para os três filtros avaliados para a condição de ausência da camada filtrante (Tabela 2), confeccionaram-se as curvas apresentadas na Figura 4.

TABELA 2. Valores de perda de carga $(\mathrm{kPa})$ para os três filtros avaliados vazios para diferentes taxas de filtração $\left(\mathrm{m}^{3} \mathrm{~m}^{-2} \mathrm{~h}^{-1}\right)$ e os respectivos valores de desvio-padrão e coeficientes de variação (\%). Head loss values (kPa) for the evaluated filters for different filtration rates $\left(\mathrm{m}^{3} \mathrm{~m}^{-2} \mathrm{~h}^{-1}\right)$ with respective values of standard deviation $(\mathrm{kPa})$ and coefficient of variation $(\%)$.

\begin{tabular}{|c|c|c|c|c|c|c|c|c|c|}
\hline \multirow[b]{2}{*}{$\mathrm{Tf}$} & \multicolumn{2}{|c|}{ F1 } & \multirow[b]{2}{*}{ C.V. } & \multicolumn{3}{|c|}{$\mathrm{F} 2$} & \multicolumn{3}{|c|}{ F3 } \\
\hline & $\mathrm{H}_{\mathrm{f}}$ & $\sigma$ & & $\mathrm{H}_{\mathrm{f}}$ & $\sigma$ & C.V. & $\mathrm{H}_{\mathrm{f}}$ & $\sigma$ & C.V. \\
\hline 20 & 3.4 & 0.33 & 9.7 & 1.1 & 0.02 & 1.8 & 1.2 & 0.02 & 1.6 \\
\hline 40 & 4.1 & 0.13 & 3.2 & 1.7 & 0.07 & 4.1 & 1.6 & 0.05 & 3.1 \\
\hline 60 & 9.7 & 0.27 & 2.8 & 2.8 & 0.10 & 3.6 & 2.8 & 0.17 & 6.1 \\
\hline 80 & 15.3 & 0.04 & 0.3 & 3.9 & 0.13 & 3.3 & 4.5 & 0.29 & 6.4 \\
\hline 100 & 23.1 & 1.61 & 7.0 & 7.3 & 0.08 & 1.1 & 6.7 & 0.18 & 2.7 \\
\hline
\end{tabular}

Tf: Taxa de filtração aplicada durante os ensaios. 


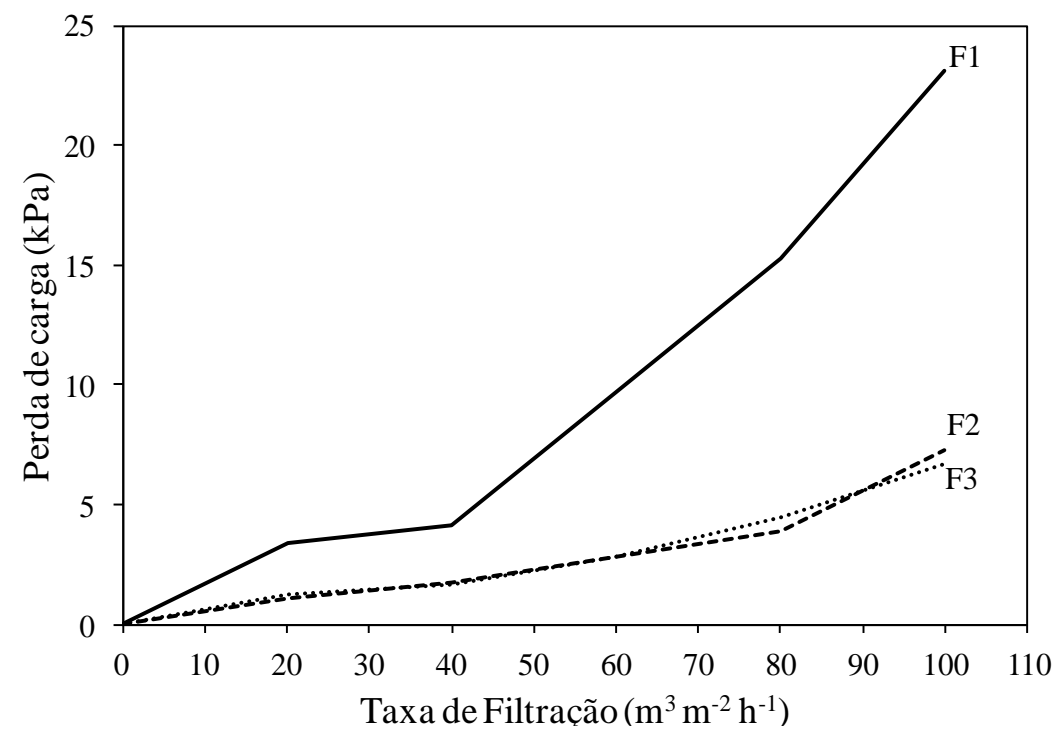

FIGURA 4. Curvas da variação da perda de carga em função da taxa de filtração para os três filtros avaliados na condição de ausência da areia em seu interior. Curves of the head loss variation as function of filtration rate for the evaluated filters for absence of sand condition.

Ao se analisar as curvas apresentadas na Figura 4, verifica-se que o filtro 1 apresentou valores significativamente maiores de perda de carga quando comparado com F2 e F3. Isso se justifica pelo fato de que F1 é constituído por um difusor diferenciado, construído com tubo de seção quadrada, que conduz o afluente até a placa difusora (Figura 3a), e drenos do tipo braço coletor, sendo que F2 e F3 utilizam crepinas e difusores dos tipos apresentados nas Figuras $3 \mathrm{~b}$ e 3c, sem tubo condutor de afluente até o difusor. $\mathrm{O}$ formato e as dimensões reduzidas da saída da seção quadrada para a placa do difusor de F1 acrescentam resistência adicional ao escoamento do afluente para dentro do corpo do filtro e, consequentemente, aumentam a dissipação de energia potencial no processo de filtragem, elevando a perda de carga da estrutura desse equipamento.

Além disso, pela Figura 4, é possível observar que F2 e F3 apresentaram comportamentos praticamente semelhantes, ou seja, proporcionaram variação idêntica da perda de carga com o incremento da taxa de filtração, sem variações significativas entre os valores encontrados para esses dois equipamentos. O comportamento hidráulico apresentado pelos filtros avaliados, sem a presença da areia em seus interiores, pode ser justificado pela diferença entre os tipos de acessórios internos desses equipamentos.

Observa-se ainda, na Figura 4, que o F1 apresentou pequena variação nos valores de perda de

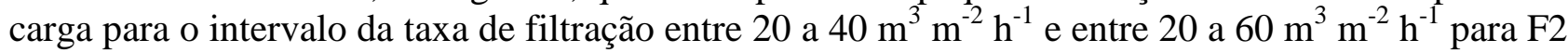
e F3, mostrando o potencial de utilização desses equipamentos dentro desses intervalos na realização da filtragem e, consequentemente, na seleção dos filtros para sistemas de irrigação que operem nessas condições. A partir dos resultados obtidos nesses ensaios com os filtros vazios, pode-se afirmar que os tipos dos componentes internos contribuem diferentemente para o comportamento da variação da perda de carga em função da taxa de filtração durante o processo de filtração.

Os ajustes dos valores médios experimentais de perda de carga, em função da vazão para cada filtro utilizando o modelo matemático exponencial, produziram equações específicas aos equipamentos avaliados, que estão apresentadas na Tabela 3, com as respectivas análises de variância, disponíveis na Tabela 4. 
TABELA 3. Equação de perda de carga em função da vazão $\left(\mathrm{m}^{3} \mathrm{~h}^{-1}\right)$ para os três filtros de areia avaliados. Head loss equations in function of the discharge $\left(\mathrm{m}^{3} \mathrm{~h}^{-1}\right)$ for three evaluated filters.

\begin{tabular}{ccc}
\hline Equipamento & Equação de Perda de Carga Unitária & $\mathrm{R}^{2}$ \\
\hline F1 & $\mathrm{H}_{\mathrm{f}}=1,934 \mathrm{e}^{0,057 \mathrm{Q}}$ & 0,963 \\
F2 & $\mathrm{H}_{\mathrm{f}}=0,688 \mathrm{e}^{0,183 \mathrm{Q}}$ & 0,991 \\
$\mathrm{~F} 3$ & $\mathrm{H}_{\mathrm{f}}=0,736 \mathrm{e}^{0,113 \mathrm{Q}}$ & 0,989 \\
\hline
\end{tabular}

TABELA 4. Análise de variância para a função exponencial característica de F1, F2 e F3. Analysis of variance for the exponential function of F1, F2 and F3.

\begin{tabular}{|c|c|c|c|c|}
\hline \multicolumn{5}{|c|}{ F1 } \\
\hline Fonte de Variação & GL & SQ & QM & $\mathrm{F}$ \\
\hline Regressão & 1 & 7,57 & 7,57 & \\
\hline Resíduo & 13 & 0,29 & 0,02 & 340,98 \\
\hline Total & 14 & 7,86 & & \\
\hline \multicolumn{5}{|c|}{ F2 } \\
\hline Fonte de Variação & GL & SQ & QM & $\mathrm{F}$ \\
\hline Regressão & 1 & 6,35 & 6,35 & \\
\hline Resíduo & 13 & 0,06 & 0,01 & $1.468,09$ \\
\hline Total & 14 & 6,40 & & \\
\hline \multicolumn{5}{|c|}{ F3 } \\
\hline Fonte de Variação & GL & SQ & QM & $\mathrm{F}$ \\
\hline Regressão & 1 & 5,80 & 5,80 & \\
\hline Resíduo & 13 & 0,06 & 0,01 & $1.234,75$ \\
\hline Total & 14 & 5,87 & & \\
\hline
\end{tabular}

GL: grau de liberdade, SQ: soma dos quadrados, QM: quadrado médio.

Observa-se que os coeficientes de determinação das três equações ajustadas se mostraram acima de $90 \%$ e com nível de significância de $1 \%$. Portanto, é possível afirmar que o modelo matemático proposto representa, significativamente, o comportamento hidráulico do processo de filtragem dos filtros de areia avaliados, para condição sem o elemento filtrante. Porém, é importante ressaltar que, para vazões com valor zero, a equação ajustada estima um valor positivo de perda de carga, mas que é próximo de zero, o que fisicamente não é possível, determinando um pequeno erro de significado físico nos ajustes, o que não o descaracteriza como aceitável para explicar esse fenômeno dentro do intervalo de operação.

Nas Figuras 5; 6 e 7 apresentam-se as curvas exponenciais ajustadas para os filtros de areia ensaiados com os respectivos valores experimentais.

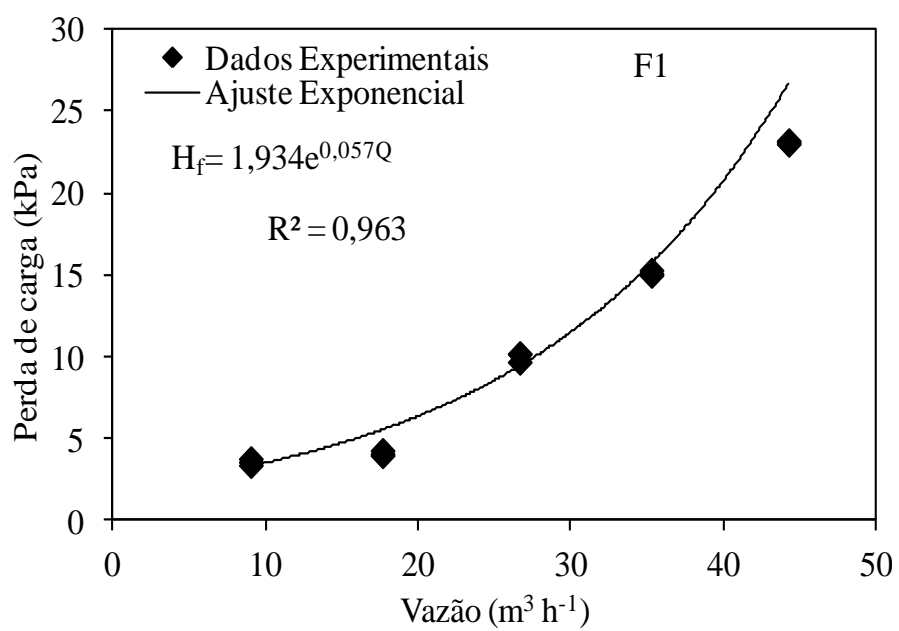

FIGURA 5. Curva característica de perda de carga em função da vazão para F1 sem camada filtrante. Characteristic curve of head loss in relation to the discharge of F1 without the filter layer. 
Pela Figura 5, correspondente a F1, pode-se observar que o menor valor de coeficiente de determinação é causado pela maior variabilidade dos dados, principalmente para o intervalo de 10 a $20 \mathrm{~m}^{3} \mathrm{~h}^{-1}$, resultante do comportamento específico desse filtro, por possuir sistema de difusão e de drenagem totalmente diferentes dos demais filtros avaliados.

Analisando-se as curvas ajustadas dos filtros ensaiados, percebe-se que o incremento da perda de carga por unidade de vazão de F2 é superior aos demais filtros ensaiados, o que pode ser comprovado pelo valor de $\beta_{1}$ nas equações. Isso pode ser explicado pelo fato de a crepina cilíndrica desse filtro ser um modelo reduzido de filtros de disco utilizados na irrigação localizada, exigindo a passagem da água filtrada pela espessura do disco, e aumentado assim a turbulência na saída do elemento. Portanto, a geometria das crepinas e as dimensões dos orifícios de saída desses componentes, bem como suas dimensões, podem causar elevação na turbulência do fluido próxima aos drenos, consequentemente, aumentando a perda de carga da estrutura.

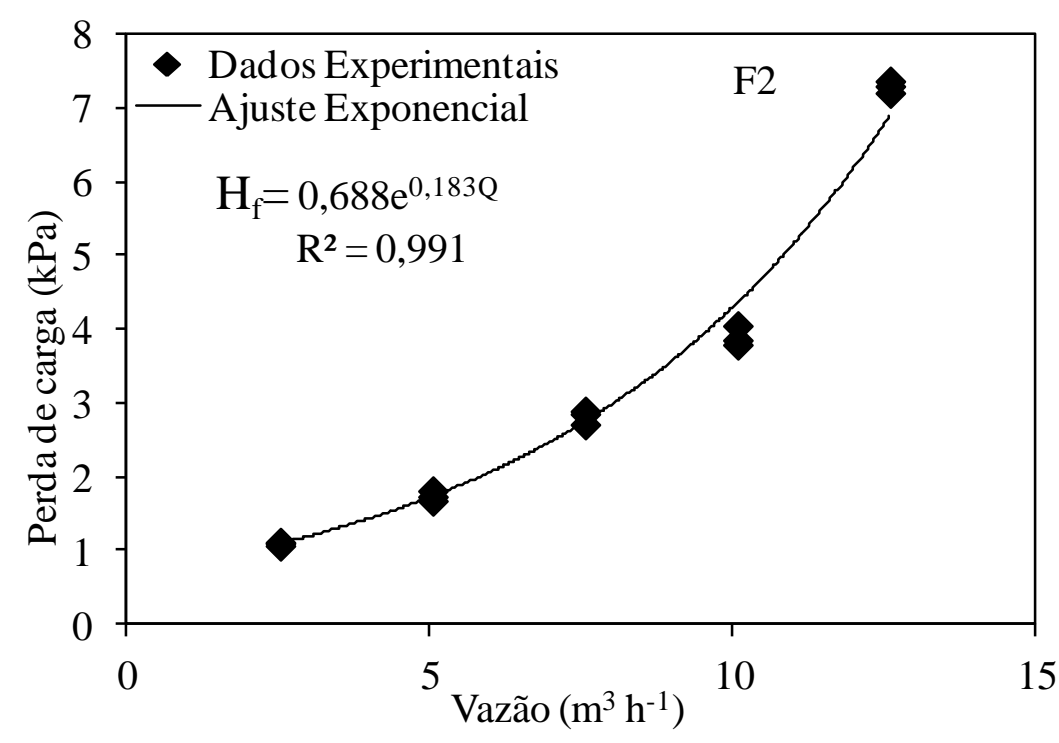

FIGURA 6. Curva característica de perda de carga em função da vazão para F2 sem camada filtrante. Characteristic curve of head loss in relation to the discharge of F2 without the filter layer.

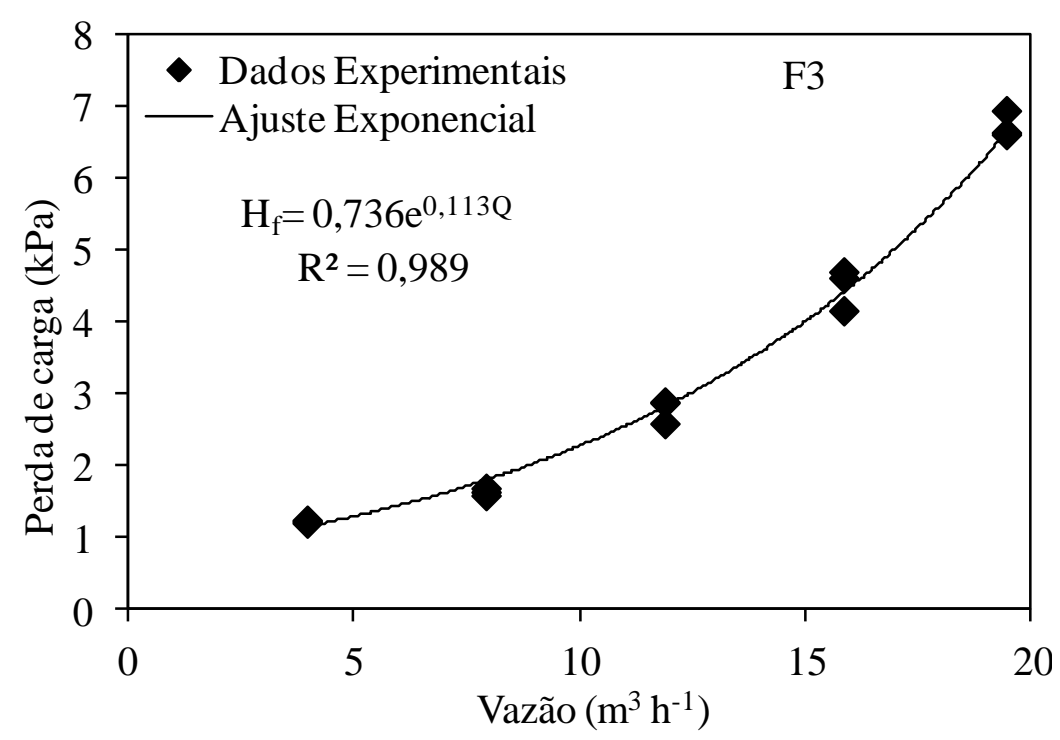

FIGURA 7. Curva característica de perda de carga em função da vazão para F3 sem camada filtrante. Characteristic curve of head loss in relation to the discharge of F2 without the filter layer. 
Pode-se afirmar que a variação de perda de carga para os filtros vazios e utilizando água limpa foi determinada somente pelo efeito dos respectivos componentes de drenagem e difusores, pois as demais características estruturais dos filtros eram semelhantes, como, por exemplo: a posição de entrada do afluente e o formato do corpo cilíndrico. O comportamento desses parâmetros foi caracterizado pela equação de cada filtro, não havendo a dissociação para cada elemento em específico. O modelo matemático utilizado neste estudo estimou a perda da carga, para os diferentes filtros de areia avaliados, com precisão aceitável, dentro dos limites das variáveis estudadas.

\section{CONCLUSÕES}

Para a condição de filtros vazios, os componentes internos do filtro 1 determinaram valores de perda de carga maiores para os mesmos valores de carga hidráulica quando comparados a F2 e F3, que apresentaram comportamentos hidráulicos idênticos. F2 apresentou maior variação de perda de carga por unidade de vazão do que os outros filtros avaliados.

A função matemática exponencial representou, significativamente, o fenômeno físico de perda de carga em função da vazão para os filtros de areia vazios utilizando água limpa.

Portanto, os tipos de estruturas internas, placa difusora e drenos, atuaram diferentemente no comportamento hidráulico dos filtros de areia, modificando o padrão de operação para cada modelo de filtro.

\section{AGRADECIMENTOS}

Às empresas Amanco do Brasil, Hidro Solo Indústria e Comércio e Marbella do Brasil, pela doação dos filtros avaliados nos ensaios.

À CAPES, pela concessão de bolsa ao primeiro autor, e ao $\mathrm{CNPq}$, pelo financiamento do projeto de pesquisa, que possibilitaram melhores condições para a realização deste trabalho.

\section{REFERÊNCIAS}

ADIN, A.; ALON, G. Mechanisms and process parameters of filter screens. Journal of Irrigation and Drainage Engineering, New York, 112, n.4, p.293-304, 1986.

ASABE. AMERICAN SOCIETY AGRICULTURAL AND BIOLOGICAL ENGINEERS. ANSI/ASAE S539. Media fFilters for irrigation-testing and performance reporting march 1994. (R2008)

ASAE STANDARDS. Design and installation of microirrigation systems. ASAE EP405.1 (DEC92). St. Joseph: American Society of Agricultural Engineers, 1993.

BENHAM, B.; ROSS, B. Filtration, treatment and maintenance: considerations for micro-irrigation systems. Virgina Cooperative Extension, 2002 (PUBLICATION, 442-757).

BURT, C.M. Media tanks for filtration - Part I: Tank Sizing and Media Selection. Irrigation Journal, p.15-17, 1994.

CHANG, M.; TRUSSELL, R.; GUZMAN, V.; MARTINEZ, J.; DELANEY, C. Laboratory studies on the clean bed head loss of filter media. Aqua, London, v.49, 1999.

MESQUITA, M. Efeito dos componentes hidráulicos e da granulometria e altura da camada filtrante na perda de carga em filtros de areia utilizados na irrigação localizada. 2010. Dissertação (Mestrado em Engenharia Agrícola) - Faculdade de Engenharia Agrícola, Universidade Estadual de Campinas, Campinas, 2010.

OLIVEIRA, C F.; FRIZZONE, J.A.; NETO, O.R. Recomendação de equações para determinação da perda de carga em filtros de tela. Revista Brasileira de Agricultura Irrigada, Fortaleza, v.1, n.1, p. 3-8, 2007. 
PHILLIPS, K.P. Long-term operation of microirrigation filtration systems: successes, failures and operational solutions. In: INTERNATIONAL MICROIRRIGATION CONGRESS, 15., 1995, Orlando. Proceddings... Orlando: ASAE, 1995. p.579-585.

PIZARRO CABELLO, F. Riegos localizados de alta frequencia. $3^{\text {rd }}$.ed. Madrid: Mundi Prensa, 1996. $513 \mathrm{p}$.

PUIG-BARGUÉS J.; RAMÍREZ DE CARTAGENA F.; BARRAGÁN J. Development of equations for calculating the head loss in effluent filtration in microirrigation systems using dimensional analysis. Biosystems Engineering, London, v.92, n,3, p.383-390, 2005

TESTEZLAF, R. Filtros de areia aplicados à irrigação localizada: teoria e prática. Engenharia Agrícola, Jaboticabal, v.28, n.3, p.604-613.

TESTEZLAF, R.; RAMOS, J.P.S. Sistemas automatizados para determinação de perda de carga em filtros de tela e disco usados na irrigação localizada. Pesquisa Agropecuária Brasileira, Brasília, v.30, p.1.079-1.088, 1995.

VERMEREIN, L.; JOBLING, G.A. Localized irrigation. Rome: FAO, 1984. 203 p. (Irrigation and Drainage Paper, 36). 